\title{
THE COHOMOLOGY ALGEBRA OF A COMMUTATIVE GROUP SCHEME
}

\author{
ROBERT FOSSUM AND WILLIAM HABOUSH
}

\begin{abstract}
Let $k$ be a commutative ring with unit of characteristic $p>0$ and let $G=\operatorname{Spec}(A)$ be an affine commutative group scheme over $k$. Let $\mathrm{H}^{\bullet}(G)$ be the graded Hochschild algebraic group cohomology algebra and, for $M$ a rational $G$-module, let $\mathrm{H}^{\bullet}(G, M)$ denote the graded Hochschild cohomology $\mathrm{H}^{\bullet}(G)$-module. We show that $\mathrm{H}^{\bullet}(G)$ is, in general, a graded Hopf algebra. When $G=\mathbf{G}_{a, k}$, let $\alpha_{p^{\nu}}$ denote the subgroup of $p^{\nu}$-nilpotents and let $\mathrm{F}_{\nu}$ denote the $\nu$ th power of the Frobenius. We show that for any finite $M$ that there is a $\nu$ such that

$$
\mathrm{H}^{\bullet}\left(\mathbf{G}_{a, k}, M\right) \cong \mathrm{H}^{\bullet}\left(\alpha_{p^{\nu}}, M\right) \otimes_{k} \mathrm{~F}_{\nu}^{*}\left(\mathrm{H}^{\bullet}\left(\mathbf{G}_{a, k}\right)\right)
$$

where $\mathrm{F}_{\nu}^{*}$ is the endomorphism of $\mathrm{H}^{\bullet}\left(\mathbf{G}_{a, k}\right)$ induced by $F_{\nu}$. As a consequence, we can show that $\mathrm{H}^{\bullet}\left(\mathbf{G}_{a, k}, M\right)$ is a finitely generated module over $\mathrm{H}^{\bullet}\left(\mathbf{G}_{a, k}\right)$ when $M$ is a finite dimensional vector space over $k$.
\end{abstract}

\section{INTRODUCTION}

Let $k$ be a commutative ring with unit and let $G=\operatorname{Spec}(A)$ be an affine group scheme over $k$. If $M$ is a $G$-module (flat over $k$ and an $A$-co-module), let $\mathrm{H}^{\bullet}(G, M)$ denote the graded $k$-module $\coprod_{n \geq 0} \mathrm{H}^{n}(G, M)$, where $\mathrm{H}^{i}(G, M)$ denotes the $i$ th Hochschild cohomology of $G$ in $M$. This is Ext in the category of rational $G$-modules (see [Demazure-Gabriel] or [Jantzen]). Then $\mathrm{H}^{\bullet}(G)$ denotes $\mathrm{H}^{\bullet}(G, k)$ for $k$ the trivial $G$-module. It is well known (loc. cit) that there is a cup product so that $\mathrm{H}^{\bullet}(G)$ is a graded algebra and $\mathrm{H}^{\bullet}(G, M)$ is a graded $\mathrm{H}^{\bullet}(G)$-module. Further $\mathrm{H}^{\bullet}(G, M)$ is contravariant in $G$ and covariant in $M$ and, if $G \rightarrow G^{\prime}$ is a morphism of group schemes, then the map $\mathrm{H}^{\bullet}\left(G^{\prime}, M\right) \rightarrow \mathrm{H}^{\bullet}(G, M)$ is compatible with the algebra morphism $\mathrm{H}^{\bullet}(G) \rightarrow$ $\mathrm{H}^{\bullet}\left(G^{\prime}\right)$. These fact are recalled and explained in the first few sections of this paper.

The main results of this paper are these: In $\S 6$, we show, for a commutative group scheme $G$, that the cohomology algebra $\mathrm{H}^{\bullet}(G)$ is canonically a graded Hopf algebra. The results of $\S \S 6$ and 7 apply to $\mathbf{G}_{a, k}$, the additive group scheme over a field $k$. Let $\alpha_{p^{\nu}, k}$ be the subgroup of $p^{\nu}$ nilpotents over $k$. Then it is known that the restriction map $\mathrm{H}^{\bullet}\left(G_{a, k}, M\right) \rightarrow \mathrm{H}^{\bullet}\left(\alpha_{p^{\nu}, k}, M\right)$ splits, though noncanonically, for sufficiently large $\nu$. Let $\mathrm{F}_{\nu}^{*}: \mathrm{H}^{\bullet}\left(\mathbf{G}_{a, k}\right) \rightarrow \mathrm{H}^{\bullet}\left(\mathbf{G}_{a, k}\right)$ be the

Received by the editors February 15, 1991 and, in revised form, May 30, 1991.

1991 Mathematics Subject Classification. Primary 14L15, 14L30, 20 G10.

Supported by the United States National Science Foundation. 
map corresponding to the $\nu$ th power of the Frobenius. In $\S 6$, we show that, for $\nu$ large enough, depending upon the finite dimensional $\mathbf{G}_{a, k}$-module $M$, there is an isomorphism:

$$
\mathbf{H}^{\bullet}\left(\mathbf{G}_{a, k}, M\right) \cong \mathrm{H}^{\bullet}\left(\alpha_{p^{\nu}, k}, M\right) \otimes_{k} \mathrm{~F}_{\nu}^{*} \mathrm{H}^{\bullet}\left(\mathbf{G}_{a, k}\right)
$$

This implies the main result of $\S 7$ which settles a long outstanding question concerning $\mathbf{G}_{a, k}$ cohomology. Namely, when $M$ is a finite dimensional representation of $\mathbf{G}_{a, k}$, then the cohomology module $\mathrm{H}^{\bullet}\left(\mathbf{G}_{a, k}, M\right)$ is a finitely generated right $\mathbf{H}^{\bullet}\left(\mathbf{G}_{a, k}\right)$-module. This question was first communicated to us by Brian Parshall in 1987. We have subsequently encountered it in conversation with several other mathematicians.

\section{Preliminaries}

Let $k$ be a commutative ring with unit and let $\operatorname{Spec}(A)=G$ be an affine group scheme over $k$. For convenience we assume that $A$ is faithfully flat over $k$. Then $A$ is a commutative Hopf algebra over $k$. Let $\mu: A \rightarrow A \otimes_{k} A$ be the co-multiplication, let $e: A \rightarrow k$ be the augmentation, and let $s: A \rightarrow A$ be the antipode.

Recall that a rational $G$-module, say $M$, is a $k$-module with a co-associative map

$$
\alpha: M \rightarrow M \otimes_{k} A
$$

such that $\left(1_{M} \otimes e\right) \circ \alpha=1_{M}$. Then the Hochschild cohomology with coefficients in $M$ is $\mathrm{H}^{i}(G, M)=\operatorname{Ext}^{i}(k, M)$ where the Ext is taken in the category of rational $G$-modules and $k$ is the trivial module. (That is: the co-action $k \rightarrow$ $k \otimes_{k} A$ is given by $a \rightarrow a \otimes 1$ for $a \in k$.)

It is well known (see [Demazure-Gabriel] or [Jantzen]) that $\mathrm{H}^{\bullet}(G, M)$ is the cohomology of the complex $\left(C^{\bullet}(G, M), \partial\right)$, where

$$
C^{q}(G, M)=M \otimes_{k} \underbrace{A \otimes A \otimes \cdots \otimes A}_{q}=M \otimes A^{\otimes q},
$$

$$
\begin{aligned}
\partial^{q}\left(m \otimes a_{1} \otimes \cdots \otimes a_{q}\right)= & \alpha(m) \otimes a_{1} \otimes \cdots \otimes a_{q} \\
& +\sum_{j=1}^{q}(-1)^{j} m \otimes a_{1} \otimes \cdots \otimes \mu\left(a_{j}\right) \otimes \cdots \otimes a_{q} \\
& +(-1)^{q+1} m \otimes a_{1} \otimes \cdots \otimes a_{q} \otimes 1 \quad(q \geq 0) .
\end{aligned}
$$

When $M=k$, write $C^{\bullet}(G)$ and $\mathrm{H}^{\bullet}(G)$ rather than $C^{\bullet}(G, k)$ and $\mathrm{H}^{\bullet}(G, k)$. Then it is again well known [Jantzen, p. 62] that

$$
C^{\bullet}(G)=k \amalg A \amalg A^{\otimes 2} \amalg \cdots \amalg A^{\otimes q} \amalg \cdots=T(A)
$$

is a ring. It is the tensor algebra of $A$ regarded as a $k$-module. 
If $u \in C^{q}(G)=A^{\otimes q}$, where $u=a_{1} \otimes \cdots \otimes a_{q}$, then

$$
\begin{aligned}
\partial^{q}(u)= & 1 \otimes a_{1} \otimes \cdots \otimes a_{q} \\
& +\sum_{j=1}^{q}(-1)^{j} a_{1} \otimes \cdots \otimes \mu\left(a_{j}\right) \otimes \cdots \otimes a_{q} \\
& +(-1)^{q+1} a_{1} \otimes \cdots \otimes a_{q} \otimes 1 .
\end{aligned}
$$

If $u_{p} \in C^{p}(G)$ and $u_{q} \in C^{q}(G)$, then

$$
\partial^{p+q}\left(u_{p} u_{q}\right)=\partial^{p}\left(u_{p}\right) u_{q}+(-1)^{p} u_{p} \partial^{q}\left(u_{q}\right) .
$$

It follows that the module of co-cycles, $Z^{\bullet}(G)=\coprod Z^{q}(G)$, is a subring of $C^{\bullet}(G)$ and that the module of co-boundaries, $B^{\bullet}(G)=\coprod B^{q}(G)$, is an ideal of $Z^{\bullet}(G)$.

Finally, it follows that $C^{\bullet}(G, M)$ is a right $C^{\bullet}(G)$-module. This is clear because $C^{\bullet}(G, M)=M \otimes_{k} C^{\bullet}(G)$. An elementary computation shows, for $u \in C^{p}(G, M)$ and $v \in C^{q}(G)$, that

$$
\partial^{p+q}(u v)=\partial^{p}(u) v+(-1)^{p} u \partial^{q}(v) .
$$

It follows immediately that $Z^{\bullet}(G, M)$ is a $Z^{\bullet}(G)$-submodule of $C^{\bullet}(G, M)$, that

$$
Z^{\bullet}(G, M) B^{\bullet}(G) \subseteq B^{\bullet}(G, M)
$$

and so that

$$
\mathrm{H}^{\bullet}(G, M)=\coprod_{r} \mathrm{H}^{r}(G, M)
$$

is a graded $\mathrm{H}^{\bullet}(G)$-module.

In general, if $M=\bigsqcup_{r} M^{r}$ is a graded module over the graded ring $S$, then $M[n]$ denotes the graded module with homogeneous components given by $M[n]^{r}=M^{r+n}$. If

$$
0 \rightarrow V^{\prime} \rightarrow V \rightarrow V^{\prime \prime} \rightarrow 0
$$

is a short exact sequence of rational $G$-modules, then the usual exact cohomology sequence becomes the exact sequence of graded $\mathrm{H}^{\bullet}(G)$-modules

$$
\cdots \rightarrow \mathrm{H}^{\bullet}\left(V^{\prime}\right) \rightarrow \mathrm{H}^{\bullet}(V) \rightarrow \mathrm{H}^{\bullet}\left(V^{\prime \prime}\right) \rightarrow \mathrm{H}^{\bullet}\left(V^{\prime}\right)[1] \rightarrow \cdots .
$$

Finally, we require some further terminology. We shall call the group scheme $G=\operatorname{Spec}(A)$ homologically exact if $C^{q}(G), B^{q}(G)$, and $\mathrm{H}^{q}(G)$ are all flat $k$ modules for all $q \geq 0$. This is true if, for example, $G$ is the base extension by $k$ of a group scheme defined over a field.

\section{Change OF GRoups}

Suppose that $G$ and $G^{\prime}$ are two affine group schemes over $k$ and that $f$ : $G \rightarrow G^{\prime}$ is a homomorphism. Let $G=\operatorname{Spec}(A), G^{\prime}=\operatorname{Spec}\left(A^{\prime}\right)$, and write $\mu, s, e, \mu^{\prime}, s^{\prime}, e^{\prime}$ for the structural data of $G$ and $G^{\prime}$ respectively. Then $f$ corresponds to a Hopf algebra map which we will write $f^{\circ}: A^{\prime} \rightarrow A$. Then $f^{\circ}$ commutes with co-multiplication, augmentation, and antipodes. Since the boundary $\partial^{q}$ is an alternating sum, each term of which is either an application 
of $\mu$ to one component or an inclusion $u \mapsto 1 \otimes u$, the diagram

$$
\begin{gathered}
C^{q}\left(G^{\prime}\right) \stackrel{\partial^{\prime q}}{\longrightarrow} C^{q+1}\left(G^{\prime}\right) \\
\downarrow f^{\circ \otimes q} \quad \downarrow f^{\circ \otimes(q+1)} \\
C^{q}(G) \stackrel{\partial^{q}}{\longrightarrow} C^{q+1}(G)
\end{gathered}
$$

commutes.

The sum $\bigsqcup_{r \geq 0} f^{\circ \otimes r}$ is surely a homomorphism of tensor algebras. So $f$ induces a functorial homomorphism of algebras, which will be denoted by $\mathrm{H}^{\bullet}(f)$, from $\mathrm{H}^{\bullet}\left(G^{\prime}\right)$ to $\mathrm{H}^{\bullet}(G)$.

Suppose that $M^{\prime}$ is a rational $G^{\prime}$-module with structure map $\alpha^{\prime}: M^{\prime} \rightarrow$ $M^{\prime} \otimes A^{\prime}$. Let $\alpha: M^{\prime} \rightarrow M^{\prime} \otimes A$ be the map given by $\alpha=\left(1_{M^{\prime}} \otimes f^{\circ}\right) \circ \alpha^{\prime}$. Then this is a rational $G$-module structure on $M^{\prime}$.

Definition 3.1. Let $f: G \rightarrow G^{\prime}$ be a homomorphism of affine algebraic group schemes over $k$. If $M^{\prime}$ is a rational $G^{\prime}$-module, then write $f^{\prime}\left(M^{\prime}\right)=M$ for the rational $G$-module with underlying module $M^{\prime}$ and co-action $\alpha=$ $\left(1_{M^{\prime}} \otimes f^{\circ}\right) \circ \alpha^{\prime}$.

Write $T(f)$ for the co-product $\bigsqcup_{q \geq 0} f^{\circ \otimes q}$ from $C^{\bullet}\left(G^{\prime}\right)$ to $C^{\bullet}(G)$. Since $M$ and $M^{\prime}$ share the same underlying $k$-module, there is a natural map

$$
T\left(f, M^{\prime}\right): C^{\bullet}\left(G^{\prime}, M^{\prime}\right) \rightarrow C^{\bullet}(G, M) .
$$

Namely, $C^{q}\left(G^{\prime}, M^{\prime}\right)=M^{\prime} \otimes C^{q}\left(G^{\prime}\right)=M^{\prime} \otimes A^{\prime \otimes q}$ and $C^{q}(G, M)=M \otimes$ $C^{q}(G)=M^{\prime} \otimes A^{\otimes q}$. Then $T^{q}\left(f, M^{\prime}\right)$ is just $1_{M^{\prime}} \otimes f^{\otimes q}$.

The map $T\left(f, M^{\prime}\right)$ is evidently a module map with respect to the homomorphism of rings $T(f)$. That is, if $\beta=T\left(f, M^{\prime}\right), \phi=T(f)$, then $\beta(u a)=\beta(u) \phi(a)$. Since $T\left(f, M^{\prime}\right)$ commutes with the differential, it induces a homomorphism

$$
\mathrm{H}^{\bullet}\left(f, M^{\prime}\right): \mathrm{H}^{\bullet}\left(G^{\prime}, M^{\prime}\right) \rightarrow \mathrm{H}^{\bullet}(G, M) .
$$

Because $T\left(f, M^{\prime}\right)$ is compatible with the ring morphism $T(f)$, it follows that $\mathrm{H}^{\bullet}\left(f, M^{\prime}\right)$ is compatible with the $\mathrm{H}^{\bullet}()$-module structure on $\mathrm{H}^{\bullet}(G, M)$ and $\mathrm{H}^{\bullet}\left(G^{\prime}, M^{\prime}\right)$ respectively. This establishes the following proposition.

Proposition 3.1. Let $G=\operatorname{Spec}(A)$ and $G^{\prime}=\operatorname{Spec}\left(A^{\prime}\right)$ be affine algebraic group schemes over $k$, let $f: G \rightarrow G^{\prime}$ be a homomorphism of group schemes, let $M^{\prime}$ be a rational $G^{\prime}$-module, and let $M=f^{!} M^{\prime}$. Then

(1) the natural map $\mathrm{H}^{\bullet}(f): \mathrm{H}^{\bullet}\left(G^{\prime}\right) \rightarrow \mathrm{H}^{\bullet}(G)$ is a homomorphism of $k$ algebras and

(2) the map $\mathrm{H}^{\bullet}\left(f, M^{\prime}\right): \mathrm{H}^{\bullet}\left(G^{\prime}, M^{\prime}\right) \rightarrow \mathrm{H}^{\bullet}(G, M)$ is an $\mathrm{H}^{\bullet}\left(G^{\prime}\right)$-module map, where $\mathrm{H}^{\bullet}\left(G^{\prime}, M^{\prime}\right)$ is an $\mathrm{H}^{\bullet}\left(G^{\prime}\right)$-module by means of the algebra map $\mathrm{H}^{\bullet}(f)$.

\section{A DIRECT LIMIT REPRESENTATION; A KÜNNETH FORMULA}

In this section assume that $k$ is a field and that the groups involved are of finite type over $k$. If $G=\operatorname{Spec}(A)$ and $G^{\prime}=\operatorname{Spec}\left(A^{\prime}\right)$, then the algebras $A$ and $A^{\prime}$ are finitely generated over $k$. 
Lemma 4.1. Let $G=\operatorname{Spec}(A)$ be an affine algebraic group scheme of finite type over $k$. Then there is a finite dimensional $k$ vector subspace $E$ of $A$ containing $k$ and such that

(1) $\mu(E) \subset E \otimes_{k} E$,

(2) $s(E)=E$,

(3) $A=k[E]$; that is $A$ is generated as an algebra by $E$.

Proof. Consider first $\mathbf{G l}(n, k)$ the coordinate ring of which is

$$
k\left[x_{11}, \ldots, x_{n n}, 1 / \delta\right],
$$

where the $\left\{x_{i j}\right\}$ are $n^{2}$ indeterminates and $\delta=\operatorname{det}\left(x_{i j}\right)$, and which is denoted by $\widetilde{A}_{n}$. Let $y_{i j}=s\left(x_{i j}\right)$. Then $y_{r s}$ is the minor in $\left(x_{i j}\right)$ corresponding to $x_{s r}$ multiplied by $(-1)^{r+s} / \delta$. Let $\widetilde{E}_{n}$ be the linear span of the $2 n^{2}+3$ elements $1, \delta, 1 / \delta,\left\{x_{i j}\right\},\left\{y_{i j}\right\}, 1 \leq i, j \leq n$. Then

$$
\begin{aligned}
\mu(1) & =1 \otimes 1, \quad \mu\left(\delta^{ \pm 1}\right)=\delta^{ \pm 1} \otimes \delta^{ \pm 1}, \\
\mu\left(x_{i j}\right) & =\sum_{l=1}^{n} x_{i l} \otimes x_{l j}, \quad \mu\left(y_{i j}\right)=\sum_{l=1}^{n} y_{i l} \otimes y_{l j} .
\end{aligned}
$$

Clearly $\widetilde{E}_{n}$ satisfies the conditions given in the lemma.

Now suppose that $G=\operatorname{Spec}(A)$ is any affine algebraic group scheme satisfying the conditions of the lemma. Chose a faithful representation $\rho: G \hookrightarrow$ Gl $(n, k)$. This determines a surjective map $\rho^{\circ}: \tilde{A}_{n} \rightarrow A$ which is in fact a Hopf algebra morphism. Let $E=\rho^{\circ}\left(\widetilde{E}_{n}\right)$. Then $E$ satisfies the conclusion of the lemma.

Corollary 4.1. Let $G=\operatorname{Spec}(A)$ be an affine algebraic group scheme of finite type over $k$. Then there is a sequence $\left\{E^{(n)} ; n \geq 1\right\}$ of finite dimensional sub-co-algebras of $A$ each with antipode and augmentation so that

(1) $A=\bigcup_{n} E^{(n)}$ and

(2) $E^{(i)} \cdot E^{(j)} \subset E^{(i+j)}$.

Proof. Choose $E^{(1)}=E$ as in the Lemma 4.1. Set

$$
E^{(n)}=\overbrace{E^{(1)} \cdots E^{(1)}}^{n}
$$

the $n$-fold product (in $A$ ) of $E^{(1)}$ with itself. (That is: the span of degree $n$ monomials in elements in $E^{(1)}$.) Since $k \subset E^{(1)}$, it follows that

$$
E^{(1)} \subset E^{(2)} \subset \cdots \subset E^{(n)} \subset \cdots
$$

and since $E$ generates $A$ over $k$, the $E^{(n)}$ exhaust $A$. The last condition is clear.

Definition 4.1. Let $G=\operatorname{Spec}(A)$ be an affine algebraic group scheme of finite type over $k$. An ascending exhaustive filtration of $A$ by finite dimensional sstable sub-co-algebras each containing $k$ is called a co-algebraic filtration of $A$. 
Suppose now that $M$ is a finite dimensional rational $G$-module. For any basis $\left\{m_{1}, \ldots, m_{l}\right\}$ of $M$, let

$$
\alpha\left(m_{i}\right)=\sum_{j} m_{j} \otimes a_{j i}, \quad a_{j i} \in A .
$$

If $\left\{E^{(r)}\right\}_{r \geq 1}$ is a co-algebraic filtration of $A$, then all of the $a_{i j}$ are in $E^{(r)}$ for some $r$. Starting from $E^{(r)}$ for this $r$ rather than from $E^{(1)}$ if necessary, we see that for any finite rational $G$-module $M$, there is a co-algebraic filtration $\left\{E^{(\bullet)}\right\}$ so that $\alpha(M) \subset M \otimes_{k} E^{(i)}$ for each $i$. When this is so, then we will say that $M$ is $E^{(\bullet)}$-compatible.

Now the Hochschild complex differential employs only the co-action on the coefficient module, the co-multiplication, and the injection of $M \otimes A^{\otimes n}$ to $M \otimes A^{\otimes(n+1)}$ via $u \mapsto u \otimes 1$. Each of these maps carries $M \otimes\left(E^{(i)}\right)^{\otimes n}$ to $M \otimes$ $\left(E^{(i)}\right)^{\otimes(n+1)}$ when $M$ is $E^{(\bullet)}$-compatible. Define subspaces of the Hochschild cochains for $M$ by the equation

$$
C^{q}\left(E^{(i)} ; M\right)=M \otimes\left(E^{(i)}\right)^{\otimes q} .
$$

Then

$$
\partial^{q}\left(C^{q}\left(E^{(i)} ; M\right)\right) \subset C^{q+1}\left(E^{(i)} ; M\right) .
$$

Hence $C^{\bullet}\left(E^{(i)} ; M\right)$ is a subcomplex of the Hochschild complex $C^{\bullet}(G, M)$. Furthermore, if $M$ and $N$ are finite and both $E^{(\bullet)}$-compatible, then the cupproduct (which is just the tensor product) induces the map

$$
C^{q}\left(E^{(i)} ; M\right) \otimes C^{r}\left(E^{(j)} ; N\right) \rightarrow C^{q+r}\left(E^{(i+j)} ; M \otimes N\right)
$$

compatible with all the structures involved.

Write $\mathrm{H}^{\bullet}\left(E^{(i)}, M\right)$ for the cohomology groups of the complex $C^{\bullet}\left(E^{(i)} ; M\right)$. Notice that

$$
C^{\bullet}(G, M)=\underset{\vec{i}}{\lim } C^{\bullet}\left(E^{(i)} ; M\right) .
$$

The inclusions $C^{\bullet}\left(E^{(i)} ; M\right) \subset C^{\bullet}\left(E^{(i+j)} ; M\right)$ induce maps of cohomology

$$
\begin{aligned}
& \mathbf{H}^{\bullet}\left(E^{(i)}, M\right) \rightarrow \mathbf{H}^{\bullet}\left(E^{(i+j)}, M\right), \\
& \mathbf{H}^{\bullet}\left(E^{(i)}, M\right) \rightarrow \mathbf{H}^{\bullet}(G, M)
\end{aligned}
$$

so that there is induced a natural homomorphism

$$
\underset{\vec{i}}{\lim } \mathrm{H}^{\bullet}\left(E^{(i)}, M\right) \rightarrow \mathrm{H}^{\bullet}(G, M)
$$

This leads to the next lemma.

Lemma 4.2. Let $G=\operatorname{Spec}(A)$ be an affine group scheme of finite type over $k$, let $E^{(\bullet)}$ be a co-algebraic filtration of $A$, and let $M$ be a finite $E^{(\bullet)}$-compatible rational G-module. Then

$$
\lim _{\vec{i}} \mathrm{H}^{\bullet}\left(E^{(i)}, M\right) \rightarrow \mathrm{H}^{\bullet}(G, M)
$$

is an isomorphism. 
Proof. Clearly

$$
Z^{q}(G, M) \cap C^{q}\left(E^{(i)}, M\right)=Z^{q}\left(E^{(i)}, M\right) .
$$

Suppose that $u \in Z^{q}\left(E^{(i)}, M\right)$ is a co-boundary in $C^{q}(G, M)$. Then there is $u^{\prime} \in C^{q-1}(G, M)$ such that $u=\partial^{q-1}\left(u^{\prime}\right)$. There is some $j$ with $j \geq i$ such that $u^{\prime} \in C^{q-1}\left(E^{(j)}, M\right)$. Then $u$ is a co-boundary in $C^{q}\left(E^{(j)}, M\right)$. The result follows.

Suppose that $A$ is the coordinate ring of an affine algebraic group scheme of finite type over $k$ and let $E^{(\bullet)}$ be a co-algebraic filtration of $A$. Let $M$ be a finite $E^{(\bullet)}$-compatible rational $G$-module. Let $\check{E}_{i}=\operatorname{Hom}_{k}\left(E^{(i)}, k\right)$ be the linear dual of $E^{(i)}$. Then by standard duality theory $\breve{E}_{i}$ is an algebra over $k$ with unit $\left.e\right|_{E^{(i)}}$. Write $e$ for this map. The definition of the multiplication on $\check{E}_{i}$ assures that $f_{1} \cdot f_{2}=\left(f_{1} \otimes f_{2}\right) \circ \mu$ for all $f_{1}, f_{2} \in \check{E}_{i}$. In particular, if $a \in E^{(i)}$ with $\mu(a)=\sum_{j=1}^{q} a_{j}^{\prime} \otimes a_{j}^{\prime \prime}$. then $\left(f_{1} \cdot f_{2}\right)(a)=\sum_{j=1}^{q} f_{1}\left(a_{j}^{\prime}\right) f_{2}\left(a_{j}^{\prime \prime}\right)$.

Now $M$ admits a bimodule structure over $\check{E}_{i}$. If $f \in \check{E}_{i}$ and $m \in M$, then

$$
f \cdot m=\left(\left(1_{M} \otimes f\right) \circ \alpha\right)(m) \text { and } m \cdot f=f(1) m .
$$

(Recall that $k \subset E^{(i)}$.) Clearly there is a canonical identificiation

$$
C^{q}\left(E^{(i)} ; M\right)=\operatorname{Hom}_{k}\left(\check{E}_{i}^{\otimes q}, M\right) .
$$

It is just a matter of definition that after rewriting co-chains as elements in $\operatorname{Hom}_{k}\left(\check{E}_{i}^{\otimes q}, M\right)$, we may write the differential as

$$
\begin{aligned}
& \left(\partial^{q}\right)(u)\left(f_{1}, f_{2}, \ldots, f_{q+1}\right)=f_{1} u\left(f_{2}, \ldots, f_{q+1}\right) \\
& \quad+\sum_{j=1}^{q-1}(-1)^{j} u\left(f_{1}, \ldots, f_{j-1}, f_{j} \cdot f_{j+1}, \ldots, f_{q+1}\right) \\
& \quad+(-1)^{q+1} f_{q+1} u\left(f_{1}, \ldots, f_{q}\right) .
\end{aligned}
$$

This proves the following lemma.

Lemma 4.3. The complex $C^{\bullet}\left(E^{(i)} ; M\right)$ is isomorphic as a complex to the Hochschild algebra cohomology complex $C^{\bullet}\left(\check{E}_{i}, M\right)$. The cohomology groups $\mathrm{H}^{\bullet}\left(\check{E}_{i}, M\right)$ are the Hochschild algebra cohomology groups of $\check{E}_{i}$ with coeffcients in the bi-module $M$.

This lemma brings us to the main result of this section. Let $G_{1}$ and $G_{2}$ be two affine algebraic group schemes of finite type over $k$. Then their product $G_{1} \times G_{2}$ is an algebraic group scheme of finite type and the projections $p_{i}$ : $G_{1} \times G_{2} \rightarrow G_{i}$ are morphisms. If $M_{1}$ is a rational $G_{1}$-module and $M_{2}$ is a rational $G_{2}$-module, then $M_{1} \otimes_{k} M_{2}$ is a rational $G_{1} \times G_{2}$-module. We may also apply 3.1 to each projection $p_{i}$. Write $M_{i}$ also for the pull-back $p_{i}^{!}\left(M_{i}\right)$. Then, in this sense, both $M_{1}$ and $M_{2}$ are $G_{1} \times G_{2}$-modules and the tensor product gives a natural pairing from the $M_{i}$ into $M_{1} \otimes M_{2}$ over $G_{1} \times G_{2}$. Hence cup product induces natural pairings

$$
\mathrm{H}^{r}\left(G_{1}, M_{1}\right) \otimes \mathrm{H}^{s}\left(G_{2}, M_{2}\right) \rightarrow \mathrm{H}^{r+s}\left(G_{1} \times G_{2}, M_{1} \otimes M_{2}\right) .
$$


Hence there is a natural cup product map

$$
\xi_{n}: \coprod_{r+s=n} \mathrm{H}^{r}\left(G_{1}, M_{1}\right) \otimes \mathrm{H}^{s}\left(G_{2}, M_{2}\right) \rightarrow \mathrm{H}^{n}\left(G_{1} \times G_{2}, M_{1} \otimes M_{2}\right) .
$$

Theorem 4.1. Let $G_{1}$ and $G_{2}$ be two affine algebraic group schemes over $k$ and let $M_{1}$ and $M_{2}$ be finite dimensional rational modules over $G_{1}$ and $G_{2}$ repectively. Then the cup product map

$$
\xi_{n}: \coprod_{r+s=n} \mathrm{H}^{r}\left(G_{1}, M_{1}\right) \otimes \mathrm{H}^{s}\left(G_{2}, M_{2}\right) \rightarrow \mathrm{H}^{n}\left(G_{1} \times G_{2}, M_{1} \otimes M_{2}\right)
$$

is an isomorphism for each $n$.

Proof. Let $G_{i}=\operatorname{Spec}\left(A_{i}\right)$ for $i=1,2$. Let $E_{i}^{(\bullet)}$ be a co-algebraic filtration of $A_{i}$ with respect to which $M_{i}$ is compatible, again for $i=1,2$. Observe that the maps $C^{\bullet}\left(E_{i}^{(j)} ; M_{i}\right) \rightarrow C^{\bullet}\left(G_{i}, M_{i}\right)$ are functorial with respect to cup product as are the cohomology maps.

Let $E_{1,2}^{(j)}=E_{1}^{(j)} \otimes E_{2}^{(j)}$. Then $E_{1,2}^{(\bullet)}$ is a co-algebraic filtration in $A_{1} \otimes A_{2}$. Hence there is a cup-product map corresponding to each term in the filtration that makes the following diagram commute:

$$
\begin{array}{ccccc}
\amalg_{r+s=n} \mathrm{H}^{r}\left(E_{1}^{(j)}, M_{1}\right) & \otimes & \mathrm{H}^{s}\left(E_{2}^{(j)}, M_{2}\right) & \stackrel{\xi_{n}^{(j)}}{\rightarrow} & \mathrm{H}^{n}\left(E_{1,2}^{(j)}, M_{1} \otimes M_{2}\right) \\
& \downarrow & & & \downarrow \\
\amalg_{r+s=n} \mathrm{H}^{r}\left(G_{1}, M_{1}\right) & \otimes & \mathrm{H}^{s}\left(G_{2}, M_{2}\right) & \stackrel{\xi_{n}}{\longrightarrow} & \mathrm{H}^{n}\left(G_{1} \times G_{2}, M_{1} \otimes M_{2}\right) .
\end{array}
$$

By Lemma 4.3, the cohomology groups $\mathrm{H}^{\bullet}\left(E_{i}^{(j)}, M_{i}\right)$ and $\mathrm{H}^{\bullet}\left(E_{1,2}^{(j)}, M_{1} \otimes M_{2}\right)$ are Hochschild algebra cohomology groups for the finite dimensional $k$-algebras $\check{E}_{i}^{(j)}$ and $\check{E}_{1,2}^{(j)}$ with coefficients in finite bi-modules. Hence [Mac Lane, Theorem VIII, 1.2, p. 222] applies, so $\xi_{n}^{(j)}$ is an isomorphism for each $n$. Applying Lemma 4.2 and passing to the direct limit, noting that direct limit commutes with tensor product over a field, we see that $\xi_{n}$ is an isomorphism for each $n$.

Corollary 4.2. Let $G_{1}, G_{2}$ be two affine algebraic groups schemes of finite type over $k$. Then

$$
\mathrm{H}^{\bullet}\left(G_{1} \times G_{2}\right)=\mathrm{H}^{\bullet}\left(G_{1}\right) \otimes \mathrm{H}^{\bullet}\left(G_{2}\right)
$$

where the isomorphism is an isomorphism of graded algebras and the tensor product is the product of graded algebras (i.e.

$$
\alpha \otimes \beta \cdot \gamma \otimes \delta=(-1)^{\operatorname{deg}(\beta) \operatorname{deg}(\gamma)}(\alpha \gamma) \otimes(\beta \delta)
$$

where the elements are homogeneous elements.)

Proof. The theorem shows that the two groups in (11) are isomorphic. That it is an isomorphism of graded objects follows because the isomorphism sends $\alpha \otimes \beta$ to $p_{1}^{*}(\alpha) \cup p_{2}^{*}(\beta)$.

\section{Commutative group SCHEMes; THE Hopf STRUCTURE}

Suppose that $G$ is a commutative affine algebraic group. Then it is a group object in the category of $k$-group schemes. This is equivalent to the fact that 
the morphisms

$$
\mu: G \times G \rightarrow G, \quad s: G \rightarrow G, \quad \epsilon: \operatorname{Spec}(k) \rightarrow G
$$

that describe the group are group homomorphisms. Also, recall that for a graded Hopf algebra, the co-multiplication takes the Hopf algebra to its graded tensor product.

We are now prepared to state one of the main results of this paper.

Theorem 5.1. Let $G$ be a commutative affine algebraic group scheme of finite type over $k$. Then $\mathrm{H}^{\bullet}(G)$ is a graded Hopf algebra with co-multiplication $\mathrm{H}^{\bullet}(\mu)$, antipode $\mathrm{H}^{\bullet}(s)$, and augmentation $\mathrm{H}^{\bullet}(\epsilon)$. If $\phi: G \rightarrow G^{\prime}$ is a homomorphism of commutative group schemes, then $\mathrm{H}^{\bullet}(\phi): \mathrm{H}^{\bullet}\left(G^{\prime}\right) \rightarrow \mathrm{H}^{\bullet}(G)$ is a morphism of Hopf algebras.

Proof. Write $\mu^{\bullet}=\mathrm{H}^{\bullet}(\mu), s^{\bullet}=\mathrm{H}^{\bullet}(s)$, and $\epsilon^{\bullet}=\mathrm{H}^{\bullet}(\epsilon)$. By Corollary 4.2, there is a natural, graded isomorphism

$$
\xi_{\bullet}: \mathrm{H}^{\bullet}(G) \otimes_{k} \mathrm{H}^{\bullet}(G) \rightarrow \mathrm{H}^{\bullet}(G \times G) .
$$

The map is the one induced by sending $\alpha \otimes \beta$ to $p_{1}^{*}(\alpha) \cup p_{2}^{*}(\beta)$, where $p_{1}$ and $p_{2}$ are the two projections. Hence $\mu^{\bullet}$ may be viewed as a map

$$
\mu^{\bullet}: \mathrm{H}^{\bullet}(G) \rightarrow \mathrm{H}^{\bullet}(G) \otimes_{k} \mathrm{H}^{\bullet}(G) .
$$

Recall that the group axioms are:

$$
\begin{aligned}
& \mu \circ\left(\mu \times 1_{G}\right)=\mu \circ\left(1_{G} \times \mu\right), \\
& \mu \circ\left(\epsilon \times 1_{G}\right)=1_{G}=\mu \circ\left(1_{G} \times \epsilon\right),
\end{aligned}
$$

and

$$
\mu \circ\left(1_{G} \times s\right) \circ \Delta=\epsilon \circ \pi=\mu \circ\left(s \times 1_{G}\right) \circ \Delta
$$

where $\Delta: G \rightarrow G \times G$ is the diagonal and $\pi: G \rightarrow \operatorname{Spec}(k)$ is the one point projection. All of these maps, without exception, are group homomorphisms (since $G$ is commutative) and so we can apply the contravariant functor $\mathrm{H}^{\bullet}($ ) to obtain

$$
\begin{gathered}
\mathrm{H}^{\bullet}\left(\mu \times 1_{G}\right) \circ \mathrm{H}^{\bullet}(\mu)=\mathrm{H}^{\bullet}\left(1_{G} \times \mu\right) \circ \mathrm{H}^{\bullet}(\mu), \\
\mathrm{H}^{\bullet}\left(\epsilon \times 1_{G}\right) \circ \mathrm{H}^{\bullet}(\mu)=\mathrm{H}^{\bullet}\left(1_{G}\right)=\mathrm{H}^{\bullet}\left(1_{G} \times \epsilon\right) \circ \mathrm{H}^{\bullet}(\mu), \\
\mathrm{H}^{\bullet}(\Delta) \circ \mathrm{H}^{\bullet}\left(1_{G} \times s\right) \circ \mathrm{H}^{\bullet}(\mu) \\
=\mathrm{H}^{\bullet}(\pi) \circ \mathrm{H}^{\bullet}(\epsilon)=\mathrm{H}^{\bullet}(\Delta) \circ \mathrm{H}^{\bullet}\left(s \times 1_{G}\right) \circ \mathrm{H}^{\bullet}(\mu) .
\end{gathered}
$$

Each of these equations incorporates certain minor inconsistencies whose resolution we leave to the reader. For example, the axiom $\mu \circ 1_{G}=1_{G}$ should really be written $\mu \circ 1_{G} \times \epsilon \circ q=1_{G}$ where $q: G \cong G \times e$ is the isomorphism of $G$ with $G \times{ }_{k} \operatorname{Spec}(k)$.

Nonetheless, each of these equations can be interpreted as a verification of the corresponding axioms in the definition of a Hopf algebra. Rather than subject the reader to these technicalities, we will select a part of (17), which we believe 
to be the most subtle, to interpret and trust the reader to verify the remaining equations.

We will show that

$$
\mathbf{H}^{\bullet}(\Delta) \circ \mathbf{H}^{\bullet}\left(s \times 1_{G}\right) \circ \mathbf{H}^{\bullet}(\mu)=\mathbf{H}^{\bullet}(\pi) \circ \mathbf{H}^{\bullet}(e)
$$

which can be interpreted as the co-inverse axiom.

First, $\mathrm{H}^{\bullet}(\mu)=\mu^{\bullet}$ is the co-multiplication and $\mathrm{H}^{\bullet}\left(s \times 1_{G}\right)=\mathrm{H}^{\bullet}(s) \otimes \mathrm{H}^{\bullet}\left(1_{G}\right)$ $=s^{\bullet} \otimes$ id under the Künneth identification. Furthermore $\mathrm{H}^{\bullet}(\pi)$ is the map from $\mathrm{H}^{\bullet}(e)$ to $\mathrm{H}^{\bullet}(G)$. Since $\mathrm{H}^{\bullet}(e)$ is just $k$ in degree 0 , it is clear that $\mathbf{H}^{\bullet}(e)$ is the algebra inclusion $j: k \rightarrow \mathbf{H}^{\bullet}(G)$.

Consider $H^{\bullet}(\Delta)$. Since $\Delta$ is a group morphism, this gives an algebra morphism $\mathrm{H}^{\bullet}(G) \otimes \mathrm{H}^{\bullet}(G) \rightarrow \mathrm{H}^{\bullet}(G)$. Here the main problem involved is the question of sign and degree, which is addressed by the fact that the tensor product is the graded tensor product. Hence we can write $m=\mathrm{H}^{\bullet}(\Delta)$, where $m$ is cup product with a sign adjustment. Rewriting (17), now with $m$ replacing $\mathbf{H}^{\bullet}(\Delta)$, gives

$$
m \circ s^{\bullet} \otimes \mathrm{id} \circ \mu^{\bullet}=j \circ \epsilon^{\bullet} .
$$

This is the left co-inverse axiom for the Hopf algebra structure with co-multiplication $\mu^{\bullet}$, antipode $s^{\bullet}$, and augmentation $\epsilon^{\bullet}$.

This is just one of the equations in the list of defining equations for a Hopf algebra.

\section{ANOTHER KÜNNETH DECOMPOSITION}

Consider the affine algebraic group scheme $G=\mathbf{G}_{a, k}$. Thus the coordinate ring of $\mathbf{G}_{a, k}$ is $A=k[x]$. For each $\nu \geq 0$, let $A_{\nu}=k \amalg k x \amalg k x^{2} \amalg \cdots \amalg$ $k x^{p^{\nu}-1}$ and let $\mathrm{F}_{\nu}^{\circ} A=k\left[x^{p^{\nu}}\right]$. Then $\mathrm{F}_{\nu}^{\circ} A$ is a sub-Hopf algebra of $k[x]$ with all of its structures. If needed, we will write $\mathrm{F}_{\nu}^{\circ} A=k[y], y=x^{p^{\nu}}$.

Lemma 6.1. The coordinate ring of $\mathbf{G}_{a, k}=\operatorname{Spec}(A)=\operatorname{Spec}(k[x])$ is isomorphic to $A_{\nu} \otimes_{k} \mathrm{~F}_{\nu}^{\circ} A$. Furthermore, this is an isomorphism of augmented co-algebras with antipode.

Proof. The map is multiplication

$$
\begin{aligned}
A_{\nu} \otimes_{k} \mathrm{~F}_{\nu}^{\circ} A & \rightarrow A, \\
x^{r} \otimes y^{s} & \rightarrow x^{r+s p^{\nu} .}
\end{aligned}
$$

No further proof is required.

Now suppose that $V$ is a finite $\mathbf{G}_{a, k}$-module with co-module structure map $\alpha: V \rightarrow V \otimes_{k} A$. Since $V$ is finite dimensional, there is some $\nu$ such that $\alpha(V) \subset V \otimes A_{\nu}$.

Now $\mathrm{F}_{\nu}^{\circ} A$ is the coordinate ring of a finite dimensional affine algebraic group. Hence there is a co-algebraic filtration $E^{\bullet}$ such that $\mathrm{F}_{\nu}^{\circ} A=\bigcup_{i} E^{(i)}$. Finally observe that defining $\mathrm{F}_{\nu}^{\circ}: A \rightarrow A$ by $\mathrm{F}_{\nu}^{\circ}(x)=x^{p^{\nu}}$ gives the Frobenius morphism 
$\mathrm{F}_{\nu}: \mathbf{G}_{a, k} \rightarrow \mathbf{G}_{a, k}$ yielding a contravariant morphism of Hochschild complexes

$$
\mathbf{F}_{\nu}^{\circ *}: C^{n}\left(\mathbf{G}_{a, k}, k\right) \rightarrow C^{n}\left(\mathbf{G}_{a, k}, k\right)
$$

and hence a contravariant morphism of cohomology rings

$$
\mathbf{H}^{\bullet}\left(\mathbf{F}_{\nu}\right): \mathbf{H}^{\bullet}\left(\mathbf{G}_{a, k}\right) \rightarrow \mathbf{H}^{\bullet}\left(\mathbf{G}_{a, k}\right) .
$$

The image of $\mathrm{F}_{\nu}^{\circ *}$ is a complex that we will denote by $C^{\bullet}\left(\mathrm{F}_{\nu}^{\circ} A\right)$. Its $r$ th term is $\left(\mathrm{F}_{\nu}^{\circ} A\right)^{\otimes r}$. Notice that $C^{\bullet}\left(\mathrm{F}_{\nu}^{\circ} A\right)$ is closed under the cup product multiplication, which is just tensor product.

Likewise the complex $C^{\bullet}\left(A_{\nu}, V\right)$ is a subcomplex of $C^{\bullet}\left(\mathbf{G}_{a, k}, V\right)$ closed under tensor multiplication by elements of $C^{\bullet}\left(A_{\nu}, k\right)$. Furthermore, the inclusion of the subgroup of $p^{\nu}$-nilpotents, $j_{\nu}: \alpha_{p^{\nu}} \rightarrow \mathbf{G}_{a, k}$, corresponds to a surjective mapping of Hopf algebras $j_{\nu}^{\circ}: k[x] \rightarrow k[x] / x^{p^{\nu}} k[x]$ which induces an isomorphism of augmented coalgebras with antipode by restriction to $A_{\nu}$. In particular (see [Jantzen, p. 69]), $j_{\nu}^{\circ}$ induces an isomorphism of $\mathrm{H}^{\bullet}\left(A_{\nu}, V\right)$ with $\mathrm{H}^{\bullet}\left(\alpha_{p}^{\nu}, V\right)$. Since this identifies $\mathrm{H}^{\bullet}\left(\alpha_{p}^{\nu}, V\right)$ with a direct summand of $\mathrm{H}^{\bullet}\left(\mathbf{G}_{a, k}, V\right)$, it proves that $\mathrm{H}^{\bullet}\left(A_{\nu}, V\right)$ is an $\mathrm{H}^{\bullet}\left(A_{\nu}\right)$-sub-module of $\mathrm{H}^{\bullet}\left(\mathbf{G}_{a, k}, V\right)$. Since there are maps $\mathrm{H}^{\bullet}\left(A_{\nu}, V\right) \rightarrow \mathrm{H}^{\bullet}\left(G_{a, k}, V\right)$ and $\mathrm{H}^{\bullet}\left(\mathrm{F}_{\nu}^{\circ} A\right)$ $\rightarrow \mathrm{H}^{\bullet}\left(\mathbf{G}_{a, k}\right)$, the cup product yields a map

$$
\eta_{\nu}: \mathrm{H}^{\bullet}\left(A_{\nu}, V\right) \otimes \mathrm{H}^{\bullet}\left(\mathrm{F}_{\nu}^{\circ} A\right) \rightarrow \mathrm{H}^{\bullet}\left(\mathbf{G}_{a, k}, V\right) .
$$

Theorem 6.1. Let $V$ be a finite dimensional representation of $\mathbf{G}_{a, k}$ and let $A_{\nu} \subset k\left[\mathbf{G}_{a, k}\right]$ be the sub-co-algebra $A_{\nu}=k \amalg k c \amalg k x^{2} \amalg \cdots \amalg k x^{p^{\nu}-1}$. Let $\mathrm{F}_{\nu}^{*}: \mathrm{H}^{\bullet}\left(G_{a, k}\right) \rightarrow \mathrm{H}^{\bullet}\left(G_{a, k}\right)$ be the contravariant map associated to the $\nu$ th order Frobenius covering. Then the cup product induces an isomorphism

$$
\eta_{\nu}: \mathrm{H}^{\bullet}\left(A_{\nu}, V\right) \otimes \mathrm{H}^{\bullet}\left(\mathrm{F}_{\nu}^{\circ} A\right) \rightarrow \mathrm{H}^{\bullet}\left(G_{a, k}, V\right) .
$$

This is evidently a map of $\mathrm{H}^{\bullet}\left(\mathrm{F}_{\nu}^{\circ} A\right)$-modules.

Proof. We proceed just as in the proof of Theorem 4.1. Begin by observing that $\mathrm{F}_{\nu}^{*}\left(\mathrm{H}^{\bullet}\left(\mathrm{F}_{\nu}^{\circ} A\right)\right)$ is exactly the image of $\mathrm{H}^{\bullet}\left(\mathrm{F}_{\nu}^{\circ} A,\right)$ in $\mathrm{H}^{\bullet}\left(\mathbf{G}_{a, k}\right)$. Now consider the co-algebraic filtration $E^{(\bullet)}$ of $\mathrm{F}_{\nu}^{\circ} A$. Then we may write

$$
C^{\bullet}\left(\mathrm{F}_{\nu}^{\circ} A\right)=\underset{\vec{n}}{\lim } C^{\bullet}\left(E^{(n)}\right) .
$$

Consider the complexes $C^{\bullet}\left(E^{(r)}\right)$ and $C^{\bullet}\left(A_{\nu}, V\right)$. Then $\tilde{E}_{r}$, the dual of $E(r)$, and $D_{\nu}$, the dual of $A_{\nu}$, are finite $k$-algebras and Lemma 4.3 applies to show that $C^{\bullet}\left(A_{\nu}, V\right)$ and $C^{\bullet}\left(E^{(r)}\right)$ are the Hochschild algebra co-chains of respectively $D_{\nu}$ in $V$ and $\tilde{E}_{r}$ in $k$. Hence, by [Mac Lane], loc. cit, the cup product induces isomorphisms

$$
\eta_{\nu, r}: \mathrm{H}^{\bullet}\left(A_{\nu}, V\right) \otimes \mathrm{H}^{\bullet}\left(E^{(r)}\right) \rightarrow \mathrm{H}^{\bullet}\left(A_{\nu} \otimes E^{(r)}, V\right) .
$$

Taking direct limits, as in Lemma 4.2, yields an isomorphism

$$
\eta_{\nu}: \mathrm{H}^{\bullet}\left(A_{\nu}, V\right) \otimes \mathrm{H}^{\bullet}\left(\mathrm{F}_{\nu}^{\circ} A\right) \rightarrow \mathrm{H}^{\bullet}\left(A_{\nu} \otimes \mathrm{F}_{\nu}^{\circ} A, V \otimes k\right) .
$$

But by the remarks preceding the statement of the theorem, $A_{\nu} \otimes \mathrm{F}_{\nu}^{\circ} A \cong A$ as Hopf algebras and it is similarly true that $V \otimes k \cong A$ under this identification. But then the result is established. 
Corollary 6.1. Let $V$ be any finite dimensional $\mathbf{G}_{a, k}$-representation. Then for some $\nu$ with $\nu>>0$, there is an isomorphism of $\mathrm{F}_{\nu}^{*} \mathrm{H}^{\bullet}\left(\mathbf{G}_{a, k}\right)$-modules

$$
\mathrm{H}^{\bullet}\left(\alpha_{p^{\nu}}, V\right) \otimes \mathrm{F}_{\nu}^{*} \mathrm{H}^{\bullet}\left(\mathbf{G}_{a, k}\right) \rightarrow \mathrm{H}^{\bullet}\left(\mathbf{G}_{a, k}, V\right) .
$$

Proof. For some $\nu$, it follows that $\alpha(V) \subset V \otimes A_{\nu}$. Then apply the theorem and observe that $\mathrm{H}^{\bullet}\left(A_{\nu}, V\right) \cong \mathrm{H}^{\bullet}\left(\alpha_{p^{\nu}}, V\right)$.

Corollary 6.2. Let $V$ be a finite dimensional representation of $\mathbf{G}_{a, k}$. Then there is a natural isomorphism of graded Hopf algebras

$$
\mathbf{H}^{\bullet}\left(\alpha_{p^{\nu}}\right) \otimes \mathbf{F}_{\nu}^{*} \mathbf{H}^{\bullet}\left(\mathbf{G}_{a, k}\right) \cong \mathbf{H}^{\bullet}\left(\mathbf{G}_{a, k}\right)
$$

compatible with the isomorphism of Corollary 6.1.

That is: $\mathrm{H}^{\bullet}\left(\mathbf{G}_{a, k}, V\right)$ is the module $\mathbf{H}^{\bullet}\left(\alpha_{p^{\nu}}, V\right)$ with coefficients extended by $\mathrm{F}_{\nu}^{*} \mathrm{H}^{\bullet}\left(\mathbf{G}_{a, k}\right)$.

Proof. The only remark necessary to make is that $\mathrm{H}^{\bullet}\left(A_{\nu}\right)$ is easily seen to be a sub-Hopf algebra of $\mathrm{H}^{\bullet}\left(\mathbf{G}_{a, k}\right)$. the rest, which does not use this fact, is clear.

To establish that $\mathrm{H}^{\bullet}\left(A_{\nu},\right)$ is canonically a (graded) Hopf algebra, note that the commutativity of $\mathbf{G}_{a, k}$ means that $\mathbf{G}_{a, k} \times \mathbf{G}_{a, k} \rightarrow \mathbf{G}_{a, k}$ is a group morphism. Hence $A \rightarrow A \otimes A$, the co-multiplication, is a co-algebra morphism. Hence $A_{\nu} \rightarrow A_{\nu} \otimes A_{\nu}$ is a co-algebra morphism. Hence $\mathrm{H}^{\bullet}\left(A_{\nu}\right)$ maps onto a graded sub-Hopf algebra of $\mathrm{H}^{\bullet}\left(\mathbf{G}_{a, k}\right)=\mathrm{H}^{\bullet}(A)$.

\section{THE COHOMOLOGY IS FINITELY GENERATED.}

In this final section we show that the cohomology $\mathrm{H}^{\bullet}\left(\mathbf{G}_{a, k}, V\right)$ of a finitely dimensional rational $\mathbf{G}_{a, k}$-module is finitely generated as a $\mathrm{H}^{\bullet}\left(\mathbf{G}_{a, k}\right)$-module. This follows easily from the results in the last section.

By a direct computation, for example [Jantzen, Proposition 4.27, p. 70], it is seen that $\mathrm{H}^{\bullet}\left(\alpha_{p^{\nu}}\right)$ is Noetherian. It is in fact a ring of commuting polynomial variables over a finite dimensional $k$-algebra.

Proposition 7.1. Let $V$ be a finite dimensional rational $\alpha_{p^{\nu}}$-module. Then $\mathbf{H}^{\bullet}\left(\alpha_{p^{\nu}}, V\right)$ is finitely generated as a $\mathbf{H}^{\bullet}\left(\alpha_{p^{\nu}}\right)$-module.

Proof. The proof is by induction on $\operatorname{dim}_{k} V$. If $\operatorname{dim}_{k} V=1$, then $V=k$ and the result is clear. Suppose that $\operatorname{dim}_{k} V>1$. Then there is a sub- $\alpha_{p^{\nu}}$-module $V^{\prime}$ of $V$ such that $\operatorname{dim}_{k}\left(V / V^{\prime}\right)=1$, or that

$$
0 \rightarrow V^{\prime} \rightarrow V \rightarrow k \rightarrow 0
$$

is exact.

Consider the resulting exact sequence (where we are writing $\mathrm{H}^{\bullet}(V)$ for $\left.\mathbf{H}^{\bullet}\left(\alpha_{p^{\nu}}, V\right)\right)$ :

$$
\cdots \rightarrow \mathbf{H}^{\bullet}(k)[-1] \rightarrow \mathbf{H}^{\bullet}\left(V^{\prime}\right) \rightarrow \mathbf{H}^{\bullet}(V) \rightarrow \mathbf{H}^{\bullet}(k) \rightarrow \mathbf{H}^{\bullet}\left(V^{\prime}\right)[1] \rightarrow \cdots .
$$

By induction, $\mathrm{H}^{\bullet}\left(V^{\prime}\right)$ is finitely generated. As $\mathrm{H}^{\bullet}(k)$ is Noetherian, it follows that

and

$$
\operatorname{Coker}\left(\mathrm{H}^{\bullet}(k)[-1] \rightarrow \mathrm{H}^{\bullet}\left(V^{\prime}\right)\right)
$$

$$
\operatorname{Ker}\left(\mathrm{H}^{\bullet}(k) \rightarrow \mathrm{H}^{\bullet}\left(V^{\prime}\right)[1]\right)
$$

are finitely generated. Hence $\mathrm{H}^{\bullet}(V)$ is also finitely generated. 
Theorem 7.1. Let $V$ be a finite dimensional, rational $\mathbf{G}_{a, k}$-module. Then $\mathrm{H}^{\bullet}\left(\mathbf{G}_{a, k}, V\right)$ is finitely generated as a $\mathrm{H}^{\bullet}\left(\mathbf{G}_{a, k}\right)$-module.

Proof. By Corollaries 6.1 and 6.2 of the previous section, there is a $\nu$ with $\nu>>0$ such that

$$
\mathrm{H}^{\bullet}\left(\mathbf{G}_{a, k}, V\right) \cong \mathrm{H}^{\bullet}\left(\alpha_{p^{\nu}}, V\right) \otimes \mathrm{F}_{\nu}^{*}\left(\mathrm{H}^{\bullet}\left(\mathbf{G}_{a, k}, k\right)\right) .
$$

Since $\mathrm{H}^{\bullet}\left(\alpha_{p^{\nu}}, V\right)$ is of finite type, the result follows.

\section{REFERENCES}

M. Demazure and P. Gabriel, Groupes algébriques. I, Masson, Paris and North-Holland, Amsterdam, 1970.

J. C. Jantzen, Representations of algebraic groups, Academic Press, Orlando, 1987.

Saunders Mac Lane, Homology, Grundlehren. Math. Wiss., Springer-Verlag, Berlin, Göttingen, and Heidelberg, 1963.

Department of Mathematics, University of Illinois, 1409 W. Green Street, Urbana, ILLINOIS 61801-2975 